\title{
Enhancement of Atmospheric Nucleation by Highly Oxygenated Organic Molecules: A Density Functional Theory Study
}

Feng Zhao, ${ }^{1,2}$ Ya-Juan Feng, ${ }^{* 2}$ Yi-Rong Liu, ${ }^{2}$ Shuai Jiang, ${ }^{2}$ Teng Huang, ${ }^{1}$ Zi-Hang Wang, ${ }^{2}$ Cai-Xin $\mathrm{Xu}^{2}$ and Wei Huang ${ }^{* 1,2,3}$

${ }^{1}$ Laboratory of Atmospheric Physico-Chemistry, Anhui Institute of Optics \& Fine Mechanics, Chinese Academy of Sciences, Hefei, Anhui 230031, China22

${ }^{2}$ School of Information Science and Technology, University of Science and Technology of China, Hefei, Anhui 230026, China

${ }^{3}$ Center for Excellent in Urban Atmospheric Environment, Institute of Urban Environment, Chinese Academy of Sciences, Xiamen, Fujian 361021, China

*E-mail: fengyj6@ustc.edu.cn, huangwei6@ustc.edu.cn 


\section{Figure Legends}

Fig. S1 Geometries of the global minima for $(\mathrm{MGA})_{2}(\mathrm{SA})_{m}(m=0-2)$ and $(\mathrm{MGA})_{2}(\mathrm{MSA})_{n}(n=0-2)$ clusters calculated at the M06-2X/6-311++G(3df, 3pd) theory level.

\section{Fig. S1}
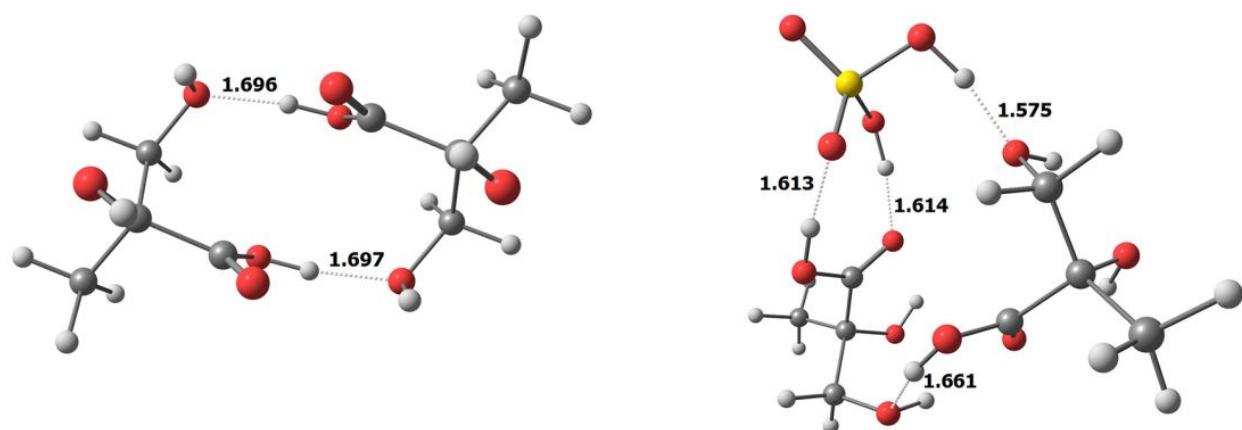

$(\mathrm{MGA})_{2}$

$(\mathrm{MGA})_{2}(\mathrm{SA})$
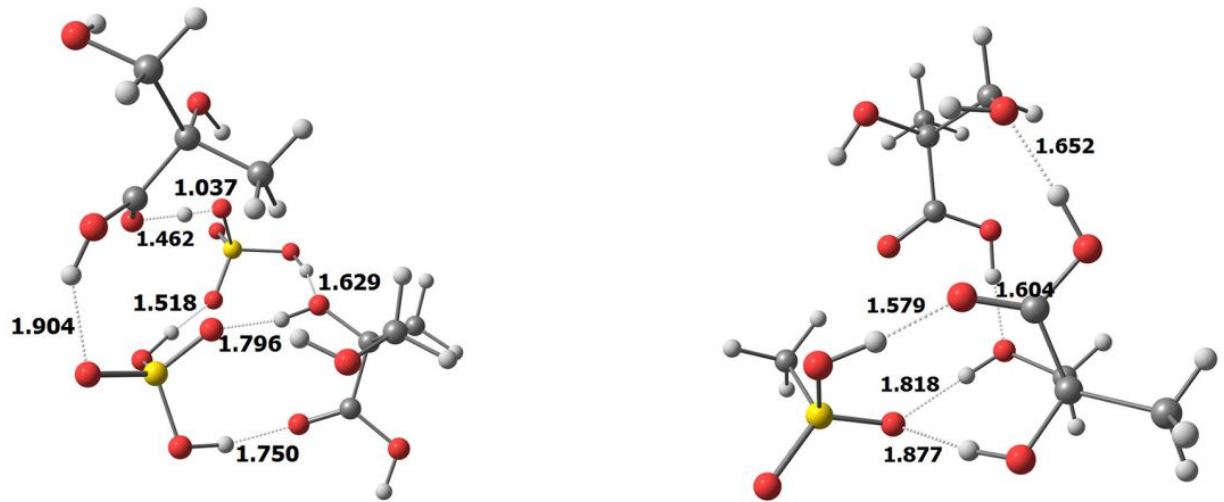

$(\mathrm{MGA})_{2}(\mathrm{SA})_{2}$

(MGA) 2 (MSA)

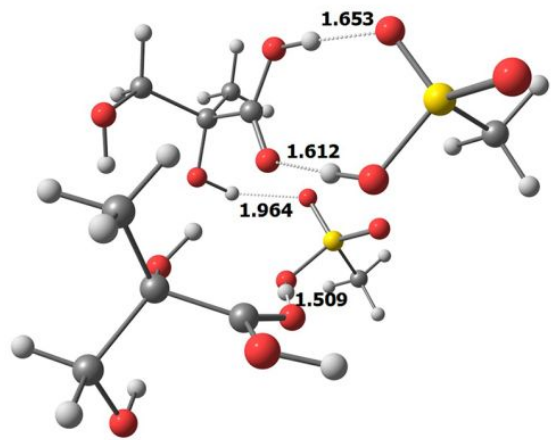

$(\mathrm{MGA})_{2}(\mathrm{MSA})_{2}$ 


\section{Table Captions}

Table S1. Binding energies $(\Delta \mathrm{E})$ and formation enthalpies $(\Delta \mathrm{H})$ calculated at DF-MP2-F12/VDZ-F12//M06-2X/6-311++G(3df,3pd) and DLPNO-CCSD(T)/aug-cc-pVTZ//M06-2X/6-311++G(3df,3pd) levels.

Table S2. Optimized Cartesian coordinates of the global minima for $(\mathrm{MGA})_{i}(\mathrm{SA})_{j}(i$ $=0-2, j=0-2)$ and $(\mathrm{MGA})_{i}(\mathrm{MSA})_{j}(i=0-2, j=0-2)$ clusters at the M06-2X/6-311++G(3df,3pd) theory level.

Table S3. Changes of entropies $(\Delta S)$ and enthalpies $(\Delta H)$ of $(\mathrm{MGA})_{i}(\mathrm{SA})_{j}(i=0-2, j$ $=0-2)$ and $(\mathrm{MGA})_{i}(\mathrm{MSA})_{j}(i=0-2, j=0-2)$ clusters at $298.15 \mathrm{~K}$.

Table S4. Total evaporation rates and formation rates of $(\mathrm{MGA})_{i}(\mathrm{SA})_{j}(i=0-2, j=$ $0-2)$ and $(\mathrm{MGA})_{i}(\mathrm{MSA})_{j}(i=0-2, j=0-2)$ clusters at $298.15 \mathrm{~K}$. The concentrations of MGA and SA/MSA were 10 pptv and $10^{7} \mathrm{~cm}^{-3}$, respectively. 
Table S1

\begin{tabular}{|c|c|c|c|c|}
\hline Clusters & \multicolumn{2}{|c|}{$\begin{array}{c}\text { DLPNO-CCSD(T)/aug-cc-pVT } \\
\mathrm{Z} / / \mathrm{M} 06-2 \mathrm{X} / 6-311++\mathrm{G}(3 \mathrm{df}, 3 \mathrm{pd})\end{array}$} & $\begin{array}{r}\text { DF-MP2-F12/VDZ-F12//M06-2 } \\
\mathrm{X} / 6-311++\mathrm{G}(3 \mathrm{df}, 3 \mathrm{pd})\end{array}$ \\
\hline & $\Delta E\left(\mathrm{kcal} \mathrm{mol}^{-1}\right)$ & $\Delta H\left(\mathrm{kcal} \mathrm{mol}^{-1}\right)$ & $\Delta E\left(\mathrm{kcal} \mathrm{mol}^{-1}\right)$ & $\Delta H\left(\mathrm{kcal} \mathrm{mol}^{-1}\right)$ \\
\hline$(\mathrm{MGA})(\mathrm{SA})$ & -15.91 & -16.42 & -16.64 & -17.16 \\
\hline$(\mathrm{MGA})(\mathrm{SA})_{2}$ & -32.52 & -32.34 & -33.25 & -33.07 \\
\hline
\end{tabular}

Table S2
MGA
$\begin{array}{llll}\text { C } & 0.282116 & 0.525187 & 0.036911\end{array}$
$\begin{array}{llll}\text { C } & 1.334438 & -0.305302 & -0.697232\end{array}$
$\begin{array}{llll}\text { C } & 0.062610 & 1.871019 & -0.647666\end{array}$
$\begin{array}{llll}\text { C } & -1.027655 & -0.248117 & 0.094194\end{array}$
$\mathrm{H} \quad 1.018405 \quad-0.492867 \quad-1.721159$
H $\quad 2.260578 \quad 0.277946 \quad-0.711971$
$\mathrm{H} \quad-0.692991 \quad 2.445004 \quad-0.113842$
$\mathrm{H} \quad 0.997046 \quad 2.428355 \quad-0.624614$
$\begin{array}{llll}\mathrm{H} & -0.253695 & 1.737661 & -1.680462\end{array}$
$\mathrm{H} \quad 0.018695 \quad 0.463984 \quad 1.953072$
H $\quad 1.719274 \quad-1.376363 \quad 0.849265$
$\mathrm{H} \quad-2.313374 \quad-1.073258 \quad-0.986202$
$\begin{array}{llll}\mathrm{O} & 0.739128 & 0.704800 & 1.357065\end{array}$
$\begin{array}{llll}\mathrm{O} & 1.514534 & -1.550052 & -0.075230\end{array}$
$\begin{array}{llll}\mathrm{O} & -1.488982 & -0.585383 & -1.110737\end{array}$
$\begin{array}{llll}\text { O } & -1.597555 & -0.502763 & 1.118735\end{array}$

\section{SA}

S $\quad-0.000054 \quad-0.000106 \quad-0.154234$

$\begin{array}{llll}\mathrm{O} & 0.637014 & -1.070886 & -0.819249\end{array}$

$\begin{array}{llll}\mathrm{O} & -0.638602 & 1.069048 & -0.820451\end{array}$

$\begin{array}{llll}\mathrm{O} & -1.030214 & -0.660033 & 0.837489\end{array}$

$\begin{array}{llll}\mathrm{O} & 1.031756 & 0.661829 & 0.834415\end{array}$

$\begin{array}{llll}\mathrm{H} & -1.686810 & -0.003834 & 1.105943\end{array}$

H $\quad 1.688051 \quad 0.005861 \quad 1.104161$

MSA

$\begin{array}{llll}\text { C } & 1.599896 & -0.360853 & -0.025816\end{array}$

$\mathrm{H} \quad 1.904813 \quad-0.334461 \quad-1.067173$ 


$\begin{array}{llll}\mathrm{H} & 2.164666 & 0.360392 & 0.558215 \\ \mathrm{H} & 1.695429 & -1.356703 & 0.394450 \\ \mathrm{H} & -1.292639 & -1.552977 & -0.321930 \\ \mathrm{O} & -0.225163 & 1.393623 & -0.573993 \\ \mathrm{O} & -0.781309 & -0.947432 & -0.873375 \\ \mathrm{O} & -0.577107 & -0.084751 & 1.388999 \\ \mathrm{~S} & -0.087688 & 0.134834 & 0.066143\end{array}$

$\begin{array}{llll}\text { (MGA)(SA) } & & \\ \mathrm{C} & -2.652233 & -0.340591 & 0.083396 \\ \mathrm{C} & -3.219466 & 0.877352 & -0.647493 \\ \mathrm{C} & -3.189405 & -1.640908 & -0.509055 \\ \mathrm{C} & -1.136060 & -0.322446 & -0.025996 \\ \mathrm{H} & -2.941573 & 0.847167 & -1.698546 \\ \mathrm{H} & -4.309518 & 0.828689 & -0.564699 \\ \mathrm{H} & -2.766056 & -2.498195 & 0.011881 \\ \mathrm{H} & -4.269204 & -1.657715 & -0.376477 \\ \mathrm{H} & -2.951802 & -1.713665 & -1.568285 \\ \mathrm{H} & -2.237248 & -0.370596 & 1.980376 \\ \mathrm{H} & -2.920366 & 2.068106 & 0.831063 \\ \mathrm{H} & 0.301262 & -0.323768 & -1.266709 \\ \mathrm{O} & -3.018410 & -0.216776 & 1.437048 \\ \mathrm{O} & -2.707736 & 2.066720 & -0.107594 \\ \mathrm{O} & -0.688128 & -0.322360 & -1.245830 \\ \mathrm{O} & -0.434934 & -0.337623 & 0.975388 \\ \mathrm{~S} & 2.815355 & -0.056217 & -0.066400 \\ \mathrm{O} & 1.973487 & -0.358098 & -1.184367 \\ \mathrm{O} & 4.145979 & -0.525423 & -0.020241 \\ \mathrm{O} & 2.823335 & 1.517491 & -0.005261 \\ \mathrm{O} & 2.104444 & -0.465997 & 1.229053 \\ \mathrm{H} & 3.552290 & 1.820217 & 0.552218 \\ \mathrm{H} & 1.095222 & -0.404670 & 1.130900 \\ & & & \\ \text { (MGA)(SA) } & & \\ \mathrm{C} & -4.420025 & -0.419747 & -0.385983 \\ \mathrm{C} & -5.041595 & -0.477136 & 1.010040 \\ \mathrm{C} & -5.228586 & 0.480209 & -1.317183 \\ \mathrm{C} & -3.001148 & 0.113698 & -0.273864 \\ \mathrm{H} & -5.083881 & 0.519331 & 1.443551 \\ \mathrm{H} & -6.059797 & -0.861705 & 0.897784 \\ \mathrm{H} & -4.762570 & 0.525614 & -2.300213 \\ \mathrm{H} & -6.222651 & 0.051941 & -1.428502 \\ \mathrm{H} & -5.309956 & 1.487053 & -0.912895 \\ \mathrm{H} & -3.498201 & -1.911851 & -1.223816\end{array}$




\begin{tabular}{|c|c|c|c|}
\hline & 213096 & 1641 & \\
\hline & -1.976175 & 1.594761 & \\
\hline & -4.379625 & -1.739269 & -0.874215 \\
\hline & -4.271586 & & \\
\hline & -2.912 & 1.28 & 0.27 \\
\hline & -2.054667 & -0.52 & -0.70 \\
\hline & 0.779 & 1.16 & 0.10 \\
\hline & 2.004 & 1.75 & -0.3 \\
\hline & -0.3 & & \\
\hline & 0.406 & 0.08 & -0.9 \\
\hline & 1.01 & 0.40 & 1.42 \\
\hline & & & \\
\hline & & -0.0 & \\
\hline & & -0.6 & \\
\hline & & -0.7 & \\
\hline & & & \\
\hline & & & \\
\hline & 4.44 & 0.78 & -0.4 \\
\hline & & & $-1 . \varepsilon$ \\
\hline & 3.54 & & -0.4 \\
\hline \multicolumn{4}{|c|}{ (MGA)(MSA) } \\
\hline & & & \\
\hline & -2.6 & 1.1 & -0.6 \\
\hline & -3.4 & -1.2 & \\
\hline & -1.1 & -0.6 & \\
\hline & & & \\
\hline & -3.6 & 1.5 & -0.5 \\
\hline & -3.3 & -2.1 & -0 \\
\hline & & & \\
\hline & -3.1 & & \\
\hline & -2.23 & -0.3 & 1.9 \\
\hline & -1.98 & 2.1 & 0.8 \\
\hline & 0.35 & -0.9 & -1. \\
\hline & -2.94 & 0.0 & 1.4 \\
\hline & -1.74 & 2.06 & -0.0 \\
\hline & -0.63 & -0.7 & -1 \\
\hline & -0.45 & -0.7 & \\
\hline & 1.972207 & 1.567535 & -0.246 \\
\hline & 2.398 & & 0.532 \\
\hline & 2.274 & 1.916695 & -1.22 \\
\hline & 0.885933 & 1.529509 & -0.170440 \\
\hline & 1.173369 & -0.694132 & 1.322731 \\
\hline & 4.044012 & -0.010229 & -0.041122 \\
\hline
\end{tabular}




\begin{tabular}{|c|c|c|c|}
\hline & 2.143101 & .474695 & \\
\hline & 1.972601 & -0.907378 & -1.026982 \\
\hline & 2.628060 & -0.055993 & -0.056686 \\
\hline \multicolumn{4}{|c|}{$(\mathrm{MGA})(\mathrm{MSA})_{2}$} \\
\hline & 2.778330 & -1.283799 & 0.038401 \\
\hline & 3.349258 & 0.074181 & 0.424804 \\
\hline & 3.32 & $-2.3^{\prime}$ & 0.95 \\
\hline & 1.251987 & -1.294566 & 0.110759 \\
\hline & 2.999302 & 0.37 & 1.40 \\
\hline & 4.438 & 0.00 & 0.4 \\
\hline & 2.906567 & -3.3 & 896 \\
\hline & 4.404834 & -2.4 & 417 \\
\hline & 3.06 & -2.1 & 1.9 \\
\hline & 2.38 & -1.8 & 449 \\
\hline & 2.96 & $0.6^{\prime}$ & -1.3 \\
\hline & -0.223346 & -0.8 & 1.200269 \\
\hline & 3.152734 & -1.5 & 912 \\
\hline & 2.94 & 1.0 & -0.5 \\
\hline & 0.769455 & -0.8 & 1.2 \\
\hline & 0.58 & -1.7 & -0.7 \\
\hline & -2.50 & -2.7 & -0 \\
\hline & -2.76 & -3 & \\
\hline & -3.15 & $-2 . \varepsilon$ & 7853 \\
\hline & -1.457497 & -2.7 & 4287 \\
\hline & -1.538779 & & -1. \\
\hline & -1.860546 & -0 . & 1.1 \\
\hline & -2.416203 & -0.2 & 9589 \\
\hline 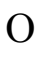 & -4.176471 & -0.9 & 128 \\
\hline & -2.792924 & -1.0 & \\
\hline & -0.941989 & 2.2 & 1.4 \\
\hline & -0.857302 & $3.1 \mathrm{c}$ & 2.005 \\
\hline & -1.983396 & & 1.3 \\
\hline & -0.398858 & 1.4 & 1.9 \\
\hline$\theta$ & 1.864643 & 2.08 & -0.117403 \\
\hline$U$ & -0.906444 & & -0.758023 \\
\hline & 1.215835 & 2.80 & 0.182083 \\
\hline $\mathrm{O}$ & -0.234309 & 1.149581 & -0.794180 \\
\hline $\mathrm{S}$ & -0.255265 & 2.436105 & -0.118445 \\
\hline \multicolumn{4}{|c|}{$(\mathrm{MGA})_{2}(\mathrm{SA})$} \\
\hline $\mathrm{C}$ & -2.988237 & -1.136947 & 0.259391 \\
\hline & -3.626392 & -0.345175 & -0.875246 \\
\hline & -3.534699 & -2.562459 & 0.309522 \\
\hline
\end{tabular}




\begin{tabular}{|c|c|c|c|}
\hline & -1.476341 & & \\
\hline & -3.419390 & -0.808635 & -1.836022 \\
\hline & -4.704940 & -0.321038 & -0.707372 \\
\hline & -3.069429 & -3.109796 & 7430 \\
\hline & -4.606608 & -2.516735 & 0.491353 \\
\hline & -3.346031 & -3.086986 & \\
\hline & -2.461679 & -0.375606 & \\
\hline & -3.12 & 1.32 & \\
\hline & -0.094413 & -1.544069 & -1.185706 \\
\hline & -3.27 & -0.4 & 1.4 \\
\hline & -3.10 & 0.96 & \\
\hline & -1.0 & -1.5 & \\
\hline & -0.7 & & 403 \\
\hline & 0.95 & 2.95 & 0.01 \\
\hline & 2.05 & 34 & \\
\hline & 0.99 & 4.10 & -0.9 \\
\hline & -0.40 & 2.2 & -0. \\
\hline & 1.93 & 1.4 & -1.1 \\
\hline & 3.0 & 2.5 & -0.2 \\
\hline & 0.16 & 4.78 & -0.7 \\
\hline & 1.92 & 4.6 & -0.8 \\
\hline & 0.94 & 3.7 & -2.0 \\
\hline & & & \\
\hline & 2.09 & 1.49 & 1.63 \\
\hline & -1.5 & 029 & -1.2 \\
\hline & & & \\
\hline & 2.15 & 1.01 & 0.79 \\
\hline & -0.5 & 1.6 & -1.2 \\
\hline & -1.22 & 2.3 & \\
\hline & 2.36 & -2.09 & -0.1 \\
\hline & 2.98 & -3.3 & -0. \\
\hline 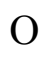 & 1.51 & -1.44 & -1.1 \\
\hline & 1.53 & -2.26 & 1.1 \\
\hline & 3.480004 & -1.10 & 0.2 \\
\hline$H$ & 0.722910 & -1.68 & 1.10 \\
\hline & 3.062510 & -0.234231 & 0.502338 \\
\hline \multicolumn{4}{|c|}{$(\mathrm{MGA})_{2}(\mathrm{SA})_{2}$} \\
\hline $\mathrm{C}$ & 2.780373 & 0.023483 & 1.515700 \\
\hline & 2.252659 & -1.102708 & 2.419974 \\
\hline & 3.570932 & 1.048498 & 2.311104 \\
\hline $\mathrm{C}$ & 3.620565 & -0.643748 & 0.432725 \\
\hline & 3.073622 & -1.508601 & 3.008658 \\
\hline & 1.519722 & -0.650205 & 3.095252 \\
\hline
\end{tabular}




$\begin{array}{llll}\mathrm{H} & 3.918081 & 1.847702 & 1.658288 \\ \mathrm{H} & 2.919977 & 1.478512 & 3.070888 \\ \mathrm{H} & 4.427665 & 0.587198 & 2.793923 \\ \mathrm{H} & 1.290707 & 0.147650 & 0.209562 \\ \mathrm{H} & 0.980368 & -1.868024 & 1.136220 \\ \mathrm{H} & 5.221874 & -1.587318 & 0.190078 \\ \mathrm{O} & 1.696395 & 0.691655 & 0.909358 \\ \mathrm{O} & 1.716061 & -2.168362 & 1.683863 \\ \mathrm{O} & 4.765940 & -1.117054 & 0.901742 \\ \mathrm{O} & 3.277036 & -0.768748 & -0.715056 \\ \mathrm{C} & -2.937640 & -0.337154 & 1.357642 \\ \mathrm{C} & -4.136827 & -1.137913 & 1.857745 \\ \mathrm{C} & -1.661691 & -0.689350 & 2.123694 \\ \mathrm{C} & -2.683410 & -0.643408 & -0.119462 \\ \mathrm{H} & -3.961222 & -2.202627 & 1.729761 \\ \mathrm{H} & -4.250367 & -0.918345 & 2.923546 \\ \mathrm{H} & -0.831603 & -0.070485 & 1.776130 \\ \mathrm{H} & -1.827379 & -0.495489 & 3.182175 \\ \mathrm{H} & -1.402640 & -1.738162 & 1.983602 \\ \mathrm{H} & -2.562316 & 1.599298 & 1.368118 \\ \mathrm{H} & -5.421581 & 0.140588 & 1.201611 \\ \mathrm{H} & -2.397435 & -2.092137 & -1.291531 \\ \mathrm{O} & -3.303653 & 1.007461 & 1.530412 \\ \mathrm{O} & -5.292415 & -0.810524 & 1.132892 \\ \mathrm{O} & -2.782215 & -1.912472 & -0.410741 \\ \mathrm{O} & -2.347254 & 0.193073 & -0.941557 \\ \mathrm{~S} & -0.031632 & 2.865551 & -0.766236 \\ \mathrm{O} & -0.452574 & 4.101801 & -1.293675 \\ \mathrm{O} & 0.552603 & 1.895284 & -1.664861 \\ \mathrm{O} & 0.986544 & 3.138080 & 0.358893 \\ \mathrm{O} & -1.165382 & 2.163382 & 0.009696 \\ \mathrm{H} & 1.374957 & 2.268972 & 0.656992 \\ \mathrm{H} & -1.663433 & 1.403667 & -0.490590 \\ \mathrm{~S} & 0.186520 & -1.499172 & -1.941835 \\ \mathrm{O} & 0.199155 & -1.047235 & -0.569845 \\ \mathrm{O} & -0.833305 & -2.407500 & -2.330204 \\ \mathrm{O} & 0.164282 & -0.290895 & -2.872116 \\ & 1.536872 & -2.191374 & -2.240325 \\ \mathrm{H} & 0.273387 & 0.592129 & -2.392477 \\ & & 2.332740 & 1.536041\end{array}$




\begin{tabular}{|c|c|c|c|}
\hline $\mathrm{C}$ & -1.344634 & 3.957699 & -0.335695 \\
\hline $\mathrm{C}$ & -0.117798 & 1.784383 & -0.684944 \\
\hline $\mathrm{H}$ & -0.178034 & 2.957835 & 1.824587 \\
\hline $\mathrm{H}$ & -1.919532 & 2.673783 & 2.053919 \\
\hline $\mathrm{H}$ & -1.584882 & 4.060160 & -1.391720 \\
\hline $\mathrm{H}$ & -2.143269 & 4.420318 & 0.241436 \\
\hline $\mathrm{H}$ & -0.403753 & 4.459764 & -0.130952 \\
\hline $\mathrm{H}$ & -2.592060 & 1.011293 & -0.027161 \\
\hline $\mathrm{H}$ & -1.353728 & 0.367311 & 1.637357 \\
\hline $\mathrm{H}$ & 1.737394 & 1.891982 & -1.005240 \\
\hline $\mathrm{O}$ & -2.500196 & 1.917375 & -0.351878 \\
\hline $\mathrm{O}$ & -0.688432 & 1.011882 & 1.933205 \\
\hline $\mathrm{O}$ & 1.001759 & 2.463189 & -0.651714 \\
\hline $\mathrm{O}$ & -0.192856 & 0.680334 & -1.187100 \\
\hline $\mathrm{C}$ & 3.182526 & -0.986617 & 0.052538 \\
\hline $\mathrm{C}$ & 3.777503 & 0.293877 & -0.516639 \\
\hline $\mathrm{C}$ & 4.106003 & -1.591179 & 1.106622 \\
\hline $\mathrm{C}$ & 1.813442 & -0.727430 & 0.682337 \\
\hline $\mathrm{H}$ & 3.907806 & 1.038312 & 0.264130 \\
\hline $\mathrm{H}$ & 4.747665 & 0.054497 & -0.957464 \\
\hline $\mathrm{H}$ & 3.677471 & -2.516481 & 1.487831 \\
\hline $\mathrm{H}$ & 5.066887 & -1.818173 & 0.648363 \\
\hline $\mathrm{H}$ & 4.253180 & -0.899764 & 1.934864 \\
\hline $\mathrm{H}$ & 2.158015 & -2.312632 & -0.906525 \\
\hline $\mathrm{H}$ & 2.611040 & 0.129779 & -2.058492 \\
\hline $\mathrm{H}$ & 0.824674 & 0.526401 & 1.717291 \\
\hline $\mathrm{O}$ & 3.002844 & -1.862393 & -1.037443 \\
\hline $\mathrm{O}$ & 2.922500 & 0.853121 & -1.498999 \\
\hline $\mathrm{O}$ & 1.769400 & 0.345010 & 1.433216 \\
\hline $\mathrm{O}$ & 0.890415 & -1.485828 & 0.499144 \\
\hline $\mathrm{C}$ & -1.790587 & -3.169605 & 0.524627 \\
\hline $\mathrm{H}$ & -1.744952 & -3.945903 & -0.233260 \\
\hline $\mathrm{H}$ & -0.801629 & -2.863697 & 0.847672 \\
\hline $\mathrm{H}$ & -2.409941 & -3.486031 & 1.359224 \\
\hline $\mathrm{H}$ & -1.141646 & -0.580714 & -1.240809 \\
\hline $\mathrm{O}$ & -2.546655 & -0.690184 & 0.763835 \\
\hline $\mathrm{O}$ & -1.691430 & -1.409075 & -1.391068 \\
\hline $\mathrm{O}$ & -3.865037 & -2.185720 & -0.676961 \\
\hline S & -2.592813 & -1.781990 & -0.192865 \\
\hline \multicolumn{4}{|c|}{$(\mathrm{MGA})_{2}(\mathrm{MSA})_{2}$} \\
\hline $\mathrm{C}$ & 0.581733 & 2.515900 & -1.523306 \\
\hline $\mathrm{C}$ & 1.436879 & 2.308304 & -2.777692 \\
\hline $\mathrm{C}$ & -0.186954 & 3.829361 & -1.606105 \\
\hline
\end{tabular}




$\begin{array}{llll}\mathrm{C} & -0.366695 & 1.324399 & -1.409048 \\ \mathrm{H} & 0.801022 & 2.287253 & -3.660671 \\ \mathrm{H} & 2.114565 & 3.164475 & -2.844595 \\ \mathrm{H} & -0.782816 & 3.976265 & -0.707527 \\ \mathrm{H} & 0.532186 & 4.642331 & -1.687830 \\ \mathrm{H} & -0.844239 & 3.838617 & -2.472297 \\ \mathrm{H} & 0.994771 & 2.648245 & 0.367994 \\ \mathrm{H} & 2.646336 & 1.060216 & -1.913551 \\ \mathrm{H} & -1.932597 & 0.546630 & -2.149276 \\ \mathrm{O} & 1.486674 & 2.489490 & -0.450205 \\ \mathrm{O} & 2.134781 & 1.091852 & -2.731893 \\ \mathrm{O} & -1.351413 & 1.343572 & -2.259934 \\ \mathrm{O} & -0.183859 & 0.426161 & -0.599163 \\ \mathrm{C} & 2.397697 & -1.571169 & -0.236361 \\ \mathrm{C} & 3.724097 & -2.250164 & 0.122041 \\ \mathrm{C} & 2.017428 & -1.860467 & -1.685848 \\ \mathrm{C} & 1.337100 & -2.120861 & 0.709167 \\ \mathrm{H} & 3.643928 & -3.325400 & -0.021933 \\ \mathrm{H} & 4.479690 & -1.852510 & -0.562035 \\ \mathrm{H} & 1.075813 & -1.380291 & -1.943565 \\ \mathrm{H} & 2.788375 & -1.453164 & -2.336391 \\ \mathrm{H} & 1.927212 & -2.931676 & -1.850274 \\ \mathrm{H} & 1.789441 & 0.303787 & -0.088546 \\ \mathrm{H} & 4.033202 & -1.073938 & 1.611406 \\ \mathrm{H} & -4.927583 & -0.995485 & 0.122372 \\ \mathrm{O} & 0.338455 & -3.656331 & 1.075211 \\ \mathrm{O} & 2.607224 & -0.207392 & 0.020772 \\ \mathrm{O} & 4.062032 & -2.025213 & 1.464815 \\ \mathrm{O} & 1.070137 & -3.396305 & 0.498071 \\ \mathrm{O} & 0.770847 & -1.493609 & 1.577392 \\ \mathrm{C} & -0.795089 & 2.052863 & 3.980150 \\ \mathrm{H} & -1.751021 & 2.560996 & 4.068932 \\ \mathrm{H} & -0.763745 & 1.169111 & 4.609393 \\ \mathrm{H} & 0.023914 & 2.728496 & 4.205843 \\ \mathrm{H} & 0.807835 & -0.036054 & 1.965095 \\ \mathrm{O} & -0.620545 & 2.720147 & 1.483138 \\ \mathrm{O} & 0.780852 & 0.929459 & 2.292716 \\ \mathrm{O} & -1.622757 & 0.535569 & 2.027128 \\ \mathrm{C} & -0.639630 & 1.539392 & 2.303923 \\ \mathrm{H} & -3.915681 & -1.075845 & 0.509283 \\ \mathrm{H} & -3.513486 & -0.108977 & 0.797234 \\ \mathrm{H} & & -0.256711 \\ & & -1.86268 & -1.862651 \\ \end{array}$




$\begin{array}{llll}\mathrm{O} & -1.502796 & -1.783451 & -0.172117 \\ \mathrm{O} & -3.330989 & -3.052903 & -1.083717 \\ \mathrm{~S} & -2.926496 & -1.723916 & -0.791219 \\ & & & \\ \mathrm{CMGA})_{2} & & \\ \mathrm{C} & -2.662432 & -0.061041 & 0.147360 \\ \mathrm{C} & -2.609604 & 1.097806 & -0.841359 \\ \mathrm{C} & -3.860658 & -0.961456 & -0.141793 \\ \mathrm{C} & -1.385004 & -0.896964 & 0.085885 \\ \mathrm{H} & -2.509301 & 0.727566 & -1.858190 \\ \mathrm{H} & -3.541226 & 1.660898 & -0.749662 \\ \mathrm{H} & -3.902482 & -1.765594 & 0.590602 \\ \mathrm{H} & -4.773769 & -0.373986 & -0.062486 \\ \mathrm{H} & -3.791776 & -1.391411 & -1.139966 \\ \mathrm{H} & -2.138740 & 0.019743 & 2.004313 \\ \mathrm{H} & -1.432386 & 2.054887 & 0.375431 \\ \mathrm{H} & -0.077121 & -1.569284 & -1.076813 \\ \mathrm{O} & -2.749454 & 0.505637 & 1.434057 \\ \mathrm{O} & -1.502595 & 1.941925 & -0.583256 \\ \mathrm{O} & -0.983552 & -1.172030 & -1.138351 \\ \mathrm{O} & -0.825287 & -1.265230 & 1.089570 \\ \mathrm{C} & 2.662405 & 0.061018 & 0.147393 \\ \mathrm{C} & 2.609603 & -1.097749 & -0.841425 \\ \mathrm{C} & 3.860715 & 0.961378 & -0.141582 \\ \mathrm{C} & 1.385049 & 0.897052 & 0.085890 \\ \mathrm{H} & 2.509312 & -0.727442 & -1.858233 \\ \mathrm{H} & 3.541227 & -1.660839 & -0.749752 \\ \mathrm{H} & 3.902508 & 1.765474 & 0.590861 \\ \mathrm{H} & 4.773780 & 0.373846 & -0.062205 \\ \mathrm{H} & 3.791968 & 1.391391 & -1.139739 \\ \mathrm{H} & 2.138509 & -0.019930 & 2.004282 \\ \mathrm{H} & 1.432451 & -2.055012 & 0.375274 \\ \mathrm{H} & 0.077295 & 1.569513 & -1.076892 \\ \mathrm{O} & 2.749287 & -0.505772 & 1.434049 \\ \mathrm{O} & 1.502606 & -1.941916 & -0.583402 \\ & 0.983697 & 1.172218 & -1.138360 \\ & 0.825212 & 1.265157 & 1.089563\end{array}$
$(\mathrm{SA})_{2}$
$\begin{array}{llll}\mathrm{S} & -2.031094 & -0.082365 & 0.089461\end{array}$
$\begin{array}{llll}\mathrm{O} & -3.390823 & -0.438362 & 0.198916\end{array}$
$\begin{array}{llll}\mathrm{O} & -1.111147 & -0.385785 & 1.142646\end{array}$
$\begin{array}{llll}\mathrm{O} & -1.496533 & -0.641228 & -1.241330\end{array}$
$\begin{array}{llll}\mathrm{O} & -1.906093 & 1.474858 & -0.086631\end{array}$ 


$\begin{array}{llll}\mathrm{H} & -0.504677 & -0.566607 & -1.282188 \\ \mathrm{H} & -2.642508 & 1.807992 & -0.617114 \\ \mathrm{~S} & 2.031125 & -0.082353 & -0.089445 \\ \mathrm{O} & 3.390973 & -0.437899 & -0.198761 \\ \mathrm{O} & 1.111277 & -0.386650 & -1.142470 \\ \mathrm{O} & 1.496869 & -0.640854 & 1.241635 \\ \mathrm{O} & 1.905471 & 1.474880 & 0.085981 \\ \mathrm{H} & 0.505084 & -0.566155 & 1.282700 \\ \mathrm{H} & 2.641645 & 1.808559 & 0.616461 \\ & & & \\ \mathrm{CMSA})_{2} & & \\ \mathrm{C} & 1.840695 & 1.628825 & 0.004312 \\ \mathrm{H} & 2.442547 & 1.985804 & 0.834068 \\ \mathrm{H} & 2.211879 & 2.017903 & -0.939871 \\ \mathrm{H} & 0.788081 & 1.871238 & 0.134516 \\ \mathrm{H} & 0.485260 & -0.609424 & 1.330048 \\ \mathrm{O} & 3.381971 & -0.470436 & -0.147871 \\ \mathrm{O} & 1.478250 & -0.596252 & 1.312921 \\ \mathrm{O} & 1.118725 & -0.606097 & -1.098645 \\ \mathrm{~S} & 2.011233 & -0.120309 & -0.067908 \\ \mathrm{C} & -3.550682 & -0.266839 & 0.144406 \\ \mathrm{H} & -3.673781 & -1.340982 & 0.235326 \\ \mathrm{H} & -3.920241 & 0.247011 & 1.027161 \\ \mathrm{H} & -4.023844 & 0.116611 & -0.754072 \\ \mathrm{H} & -0.473908 & -0.728860 & -1.313329 \\ \mathrm{O} & -1.171394 & -0.524493 & 1.174124 \\ \mathrm{O} & -1.472577 & -0.696392 & -1.240796 \\ \mathrm{O} & -1.630017 & 1.489797 & -0.136102 \\ \mathrm{~S} & -1.837217 & 0.089044 & 0.045707\end{array}$


Table S3

\begin{tabular}{ccc}
\hline Clusters & Evaporation rates $\left(\mathrm{s}^{-1}\right)$ & Formation rates $\left(\mathrm{cm}^{-3} \mathrm{~s}^{-1}\right)$ \\
\hline$(\mathrm{MGA})_{2}$ & $5.45 \mathrm{e}+06$ & $6.51 \mathrm{e}-08$ \\
$(\mathrm{MGA})(\mathrm{SA})$ & $2.91 \mathrm{e}+05$ & $3.71 \mathrm{e}-01$ \\
$(\mathrm{MGA})(\mathrm{SA})_{2}$ & $5.01 \mathrm{e}+05$ & $1.15 \mathrm{e}-08$ \\
$(\mathrm{MGA})_{2}(\mathrm{SA})$ & $7.40 \mathrm{e}+05$ & $6.51 \mathrm{e}-08$ \\
$(\mathrm{MGA})_{2}(\mathrm{SA})_{2}$ & $8.21 \mathrm{e}+14$ & $8.58 \mathrm{e}-16$ \\
$(\mathrm{MGA})(\mathrm{MSA})$ & $4.00 \mathrm{e}+06$ & $3.60 \mathrm{e}-02$ \\
$(\mathrm{MGA})(\mathrm{MSA})_{2}$ & $1.50 \mathrm{e}+09$ & $4.53 \mathrm{e}-10$ \\
$(\mathrm{MGA})_{2}(\mathrm{MSA})$ & $6.26 \mathrm{e}+07$ & $3.55 \mathrm{e}-10$ \\
$(\mathrm{MGA})_{2}(\mathrm{MSA})_{2}$ & $5.67 \mathrm{e}+14$ & $1.03 \mathrm{e}-19$ \\
\hline
\end{tabular}

Table S4

\begin{tabular}{ccc}
\hline Clusters & $\left.\Delta H\left(\mathrm{kcal} \mathrm{mol}^{-1}\right)\right)$ & $\Delta S\left(\mathrm{cal} \mathrm{mol}^{-1} \mathrm{~K}^{-1}\right)$ \\
\hline$(\mathrm{SA})_{2}$ & -17.35 & -35.00 \\
$(\mathrm{MGA})(\mathrm{SA})$ & -17.16 & -36.90 \\
$(\mathrm{MGA})(\mathrm{SA})_{2}$ & -33.07 & -67.53 \\
$(\mathrm{MGA})_{2}$ & -17.60 & -44.00 \\
$(\mathrm{MGA})_{2}(\mathrm{SA})$ & -37.65 & -86.44 \\
$(\mathrm{MGA})_{2}(\mathrm{SA})_{2}$ & -43.95 & -125.30 \\
\hline$(\mathrm{MSA})_{2}$ & -18.41 & -37.52 \\
$(\mathrm{MGA})(\mathrm{MSA})$ & -16.95 & -41.27 \\
$(\mathrm{MGA})(\mathrm{MSA})_{2}$ & -32.51 & -80.83 \\
$(\mathrm{MGA})_{2}$ & -17.60 & -44.00 \\
$(\mathrm{MGA})_{2}(\mathrm{MSA})$ & -34.14 & -87.80 \\
$(\mathrm{MGA})_{2}(\mathrm{MSA})_{2}$ & -42.42 & -125.31 \\
\hline
\end{tabular}

